\title{
Putative fructose-1,6-bisphosphate aldolase 1 (AtFBA1) affects stress tolerance in yeast and Arabidopsis
}

\author{
Seok-Jun Moon $\cdot$ Dongjin Shin $\cdot$ Beom-Gi Kim $\cdot$ Myung-Ok Byun
}

Received: 1 June 2012 / Accepted: 7 June 2012

(C) Korean Society for Plant Biotechnology

\begin{abstract}
Glycolysis is responsible for the conversion of glucose into pyruvate and for supplying reducing power and several metabolites. Fructose-1,6-bisphosphate aldolase (AtFBA1), a central enzyme in the glycolysis pathway, was isolated by functional complementation of the salt-sensitive phenotype of a calcineurin $(\mathrm{CaN})$-deficient yeast mutant. Under high salinity conditions, aldolase activity and the concentration of NADH were compromised. However, expression of AtFBA1 maintained aldolase activity and the NADH level in yeast cells. AtFBAl shares a high degree of sequence identity with known class I type aldolases, and its expression was negatively regulated by stress conditions including $\mathrm{NaCl}$. The fusion protein GFP-AtFBA1 was localized in the cytosol of Arabidopsis protoplasts. The seed germination and root elongation of AtFBA1 knock-out plants exhibited sensitivity to ABA and salt stress. These results indicate that AtFBA1 expression and aldolase activity is important for stress tolerance in yeast and plants.
\end{abstract}

Keywords Fructose-1,6-bisphosphate aldolase, $\mathrm{NaCl}$, Germination assay

\section{Introduction}

In plants, unfavorable environmental conditions such as high salinity and drought result in biochemical, molecular, and physiological changes that adversely affect plant growth and development, and ultimately compromise crop productivity (Skirycz and Inze 2010; Chew and Halliday 2011). Plants have evolved sophisticated mechanisms to adapt or survive adverse environmental conditions (Chew and Halliday 2011).

S.-J. Moon · D. J. Shin • B.-G. Kim • M.-O. Byun ( $ه)$

Molecular breeding division, National Academy of Agricultural Science, RDA, Suwon, 441-857, Korea

e-mail: mobyun@rda.go.kr
The phytohormone abscisic acid (ABA) accumulates under conditions of environmental stress and activates signal transduction pathways (Chew and Halliday 2011). This leads to a variety of physiological responses including metabolic alterations and the expression of stress-related genes (Wang et al. 2011). Recently, it was reported that ABI1/PP2C along with the RCAR/PYR1/PYL family of START proteins is able to bind ABA through a gate-latch-lock mechanism in Arabidopsis (Melcher et al. 2009). Additionally, it was shown that ABA perception suppresses PP2C-mediated dephosphorylation of SnRKs and permits their activation (Melcher et al. 2009; Park et al. 2009). The pyrl/pyl1/pyl2/ pyl4 quadruple mutant shows insensitivity in ABA-induced stomatal closure and ABA-inhibited stomatal opening (Nishimura et al. 2010).

Stress conditions such as high salinity and ABA treatment restrict photosynthesis resulting in energy deprivation and growth retardation (Quan et al. 2010). This causes a disruption in the levels of metabolites resulting from normal metabolism (Rao et al. 2010). Some of these metabolites function as compatible solutes including sugars, low-complexity carbohydrates, and amino acids. Some metabolites, such as sucrose and glucose, function as signal molecules in important regulatory processes (Baier et al. 2004; Cho and Yoo 2011). In plants, glucose has emerged as a key regulator of many vital processes, including germination, seedling development, and stress responses (Jin et al. 2010; Kushwah et al. 2011). Arabidopsis HXK1 (AtHXK1) functions as an intracellular glucose sensor. At high glucose concentrations, nuclear AtHXK1 binds glucose and functions as a transcriptional repressor together with VHA-B1, a subunit of the peripheral V1 complex in vacuolar-type H-ATPase, and RPT5B, a subunit of the 19S regulatory particle (Moore et al. 2003; Cho et al. 2006). This interaction causes the repression of genes involved in photosynthesis (Cho et al. 2006). Jang et al. constructed transgenic plants expressing either sense or antisense constructs of two genes encoding hexokinase 
isoforms, Arabidopsis HEXOKINASE1 (HXKl) and HXK2. Plants overexpressing $H X K$ genes exhibited glucose-hypersensitive characteristics (Jang et al. 1997; Moore et al. 2003). By contrast, antisense plants displayed hyposensitive phenotypes (Jang et al. 1997). High levels of exogenous glucose are believed to result in ABA accumulation (Finkelstein and Gibson 2002). This, in turn, results in delayed germination and inhibited seedling development (Finkelstein and Gibson 2002; Price et al. 2003). Arenas-Huertero et al. showed that the glucose insensitive mutant 6 (gin6) mutant abolishes the glucose-induced expression of $A B I 4$ (Arenas-Huertero et al. 2000). In addition, the glucose-specific accumulation of $\mathrm{ABA}$ is compromised in the gin5 mutant (Arenas-Huertero et al. 2000). Snfl-related protein kinases (SnRKs) function within an intricate network linking metabolic and stress signaling in plants (Halford and Hey 2009). These results suggest that ABA plays a central role in the glucose signaling network that controls postembryonic development. Fructose-bisphosphate aldolase (EC 4.1.2.13) catalyzes two reactions: the aldol cleavage of fructose-1,6-bisphosphate into glyceraldehyde-3-phosphate (GAP) and dihydroxyacetonephosphate (DHAP), and the reversible aldol condensation reaction (Lorentzen et al. 2004). The enzyme functions as a constituent of both the glycolytic/gluconeogenic pathway and the pentose phosphate cycle in plants (Lorentzen et al. 2004). Fructose-bisphosphate aldolase catalyzes a readily reversible reaction, possesses no known regulatory properties, and appears irrelevant for the control of metabolism and growth. However, small changes in its activity have striking effects on photosynthesis, carbon partitioning and growth (Haake et al. 1998). Konishi et al. demonstrated that fructosebisphosphate aldolase is regulated by gibberellin in rice roots. Additionally, the study suggested that aldolase associates with vacuolar $\mathrm{H}^{+}$-ATPase in roots, potentially regulating the vacuolar $\mathrm{H}^{+}$-ATPase-mediated control of cell elongation (Konishi et al. 2004). Nonetheless, the relationship of aldolase with glycolytic enzymes and stress responses remains to be characterized. Here, AtFBAl is reported to encode a putative fructose-1,6-bisphosphate aldolase in Arabidopsis. AtFBA1 is able to suppress the salt-sensitive phenotype of $c n b \Delta$ cells and plays a role in seed germination and root growth under environmental stress conditions in plants.

\section{Materials and methods}

Complementation assay

The AtFBA1 gene was subcloned into pYES2, an expression vector containing the galactose-inducible GAL1 promoter and the URA3 selection marker. The construct was introduced into the YP9 strain (Mata ura3 leu2 his3 trp1 ade2 lys2 cnb1::HIS3), and transformed cells were selected on SD-Uramedium (Shin et al. 2004). The transformants were grown in SD-Ura- medium overnight. Aliquots $(2 \mu \mathrm{l})$ from an exponentially growing cell culture (O.D. $600=0.1)$ were serial diluted (1:10, 1:100, 1:1000) and spotted onto YPGal plates containing $\mathrm{NaCl}(1.1 \mathrm{M}), \mathrm{LiCl}(100 \mathrm{mM}), \mathrm{KCl}(1.4 \mathrm{M})$ and sorbitol $(1.5 \mathrm{M})$. Colonies were examined after 4 days of growth at $30^{\circ} \mathrm{C}$.

Enzyme assay, kinetics, and determination of NADH levels

Yeast cells were grown to a density of O.D. $600=0.1$ in YPD medium. AtFBA1 expression was examined in cells that had been washed in sterile water and resuspended in YPGal medium for 18-20 hours. The cells were collected by centrifugation at $3000 \mathrm{rpm}$ for $10 \mathrm{~min}$. Cells were washed twice with lysis buffer, resuspended in 1/10 culture volume of lysis buffer, and ruptured with glass beads. The supernatant (50 $\mu \mathrm{g}$ protein) was precipitated with $10 \%$ TCA to estimate the aldolase activity and NADH level. An aldolase cleavage assay was performed by monitoring NADH oxidation at 340 $\mathrm{nm}$ and $22^{\circ} \mathrm{C}$ through a coupled assay system with triosephosphate isomerase and glycerol-3-phosphate dehydrogenase. NADH was detected using a Genesys 10uv spectrophotometer (Thermo Spectronic, USA). The reaction was initiated by adding total protein from the transformants harboring the $A t F B A 1$ construct or the vector alone.

Northern blot analysis

Two-week-old Arabidopsis seedlings were treated with $\mathrm{NaCl}$ $(100 \mathrm{mM})$, ABA $(10 \mu \mathrm{M})$, or cold $\left(4^{\circ} \mathrm{C}\right)$ for the times indicated $(0,0.5,1,3,6$, and 12 hours). Total RNA was isolated, and $20 \mu \mathrm{g}$ was separated in a $1.5 \%$ formaldehyde agarose gel and transferred to a charged membrane. After transfer, the RNA was UV cross-linked to the membrane and used for northern blot analysis. Hybridization and washing was conducted according to a published protocol (Shin et al. 2011). The full-length AtFBAl gene was used as a probe.

In vivo targeting of AtFBA1 in Arabidopsis protoplasts

AtFBA1 cDNA was inserted into the p326-sGFP plasmid to create chimeric GFP-fusion constructs controlled by the 35S promoter. The plasmid was introduced into Arabidopsis protoplasts that had been prepared from leaf tissues by 
polyethylene glycol-mediated transformation (Shin et al. 2004). Expression of the fusion constructs was monitored at various time points after transformation, and images were captured using a cooled CCD camera and a Zeiss Axioplan fluorescence microscope (Carl Zeiss Co, Jena, Germany). Data were processed using Adobe Photoshop software (Adobe System, Mountain view, CA) and presented in pseudo-color format.

Plant material, germination assay and root growth measurements

Arabidopsis thaliana (ecotype Columbia) was grown either on MS medium or soil in a growth chamber with a 16-hour light/8-hour dark photoperiod at $22^{\circ} \mathrm{C}$ and $70 \%$ relative humidity. A mutant containing a T-DNA insertion in the AtFBAl locus was obtained from the Arabidopsis Biological Resource Center at Ohio State University.

To test the germination frequency, seeds of wild-type and knock-out plants were stratified at $4^{\circ} \mathrm{C}$ for 3 days. Seeds were plated on $1 / 2 \mathrm{MS}$ medium with or without $\mathrm{NaCl}(100$ $\mathrm{mM})$, ABA $(10 \mu \mathrm{M})$, or $\mathrm{KCl}(100 \mathrm{mM})$, and transferred to a growth chamber $\left(22^{\circ} \mathrm{C}\right.$ with a 16 -hour light/8-hour dark regime). Germination frequencies were determined by scoring radical emergence ( $\mathrm{n}=3, \sim 200$ seeds per experiment). To measure root length, seeds of wild-type and atfbal-1 plants were plated on 1/2 MS medium in a vertical orientation. Five-day-old seedlings were transferred to $1 / 2 \mathrm{MS}$ medium with or without $\mathrm{NaCl}, \mathrm{KCl}$, or mannitol. Seedlings were incubated in the growth chamber for an additional 7 days, and root lengths were measured. Three replicate plates were used for each treatment to ensure data reproducibility.

\section{Results}

Putative fructose-1,6-bisphosphate aldolase complements the salt-sensitive phenotype of the yeast $\mathrm{CaN}$ null mutant $(c n b \Delta)$.

Several plant genes able to suppress the salt-sensitive phenotype of the CaN null mutant were identified (Shin et al., 2004). One such gene, a putative fructose-1,6-bisphosphate aldolase (AtFBA1, accession no. At3g52930), was further examined. The cDNA of AtFBAl consists of 1780 nucleotides encoding a polypeptide of 358 amino acids. A comparison of the AtFBA1 amino acid sequence with other known proteins indicates that AtFBA1 shares a high degree of sequence identity with Class I type fructose-1,6-bisphosphate aldolases from rice, maize or spinach $(82.6 \%, 81.2 \%$, and
$78.7 \%$, respectively; data not shown).

To validate the screening results, the full-length AtFBAI gene was cloned into the episomal pYES2 plasmid and introduced into the yeast $c n b \Delta$ strain. Transformation with AtFBA1 complemented the salt-sensitive phenotype of the $c n b \Delta$ cells (Fig. 1). In yeast, $\mathrm{NaCl}$ is known to cause ionic and non-ionic (osmotic) stress, while $\mathrm{KCl}$ and sorbitol specifically trigger osmotic stress. To examine whether AtFBA1 expression plays a role in ionic stress tolerance or osmotic stress tolerance, the phenotype of yeast cells following $\mathrm{LiCl}, \mathrm{KCl}$ and sorbitol treatment was assessed. As shown in Figure 1, AtFBAl expression increased $\mathrm{LiCl}, \mathrm{KCl}$ and sorbitol tolerance in yeast. These results indicate that AtFBAI expression is involved in the osmotic tolerance of yeast. AtFBA1 encodes a fructose-1,6-bisphosphate aldolase, one of the enzymes involved in glycolysis. Expression of AtFBAI suppressed the salt-sensitive phenotype of $c n b \Delta$ cells. These results suggest that aldolase metabolic activity in yeast may be affected by $\mathrm{NaCl}$. To investigate this possibility, aldolase activity was examined using a coupled assay with triosephosphate isomerase (TIM) and glycerol-3-phosphate dehydrogenase (GPDH). Under conditions of salt stress, the aldolase activity of control $c n b \Delta$ cells was decreased by $40 \%$. However, when AtFBAl was expressed in $c n b \Delta$ cells, the aldolase activity increased in the presence and absence of salt treatment. When glyeraldehyde-3-phosphate (GAP) is converted into 1,3-bisphosphoglyerate (1,3-BisPGA) via GAP dehydrogenase, NADH is generated. Therefore, the concentration of NADH under high salinity conditions was examined. The NADH level of control $c n b \Delta$ cells was decreased by approximately $25 \%$. By contrast, $c n b \Delta$ cells harboring AtFBAl maintained high levels of NADH following $\mathrm{NaCl}$ treatment (Fig. 2). These data suggest that although

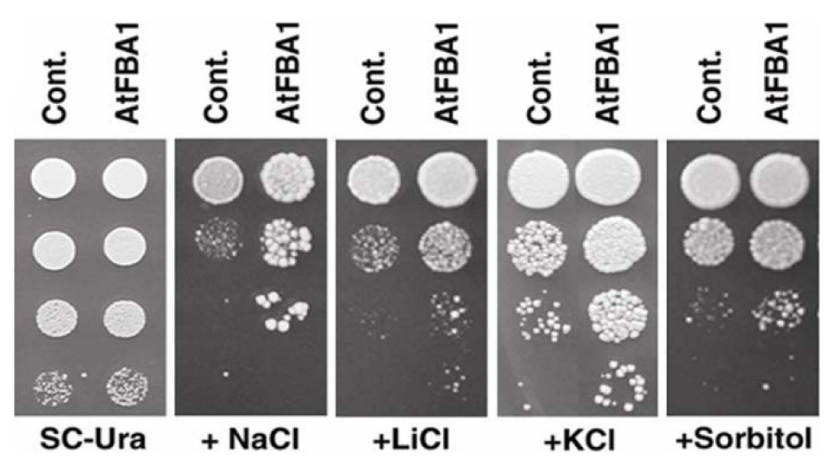

Fig. 1 Expression of AtFBA1 suppresses the salt sensitive phenotype of $c n b \Delta$. The YP9 yeast strain was transformed with the empty vector (pYES2) containing the full-length AtFBA1 cDNA. Transformed cells were tested for tolerance to $\mathrm{NaCl}, \mathrm{LiCl}, \mathrm{KCl}$, and sorbitol as described in the Materials and Methods. Plates were photographed after incubation at $30^{\circ} \mathrm{C}$ for 3 days 
A

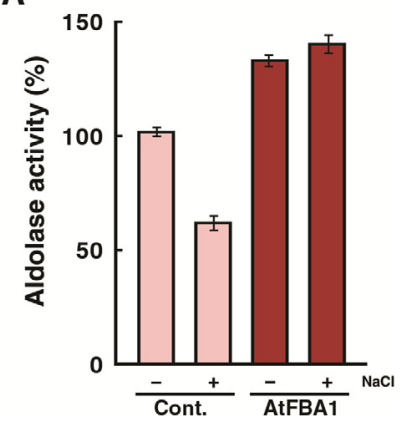

B

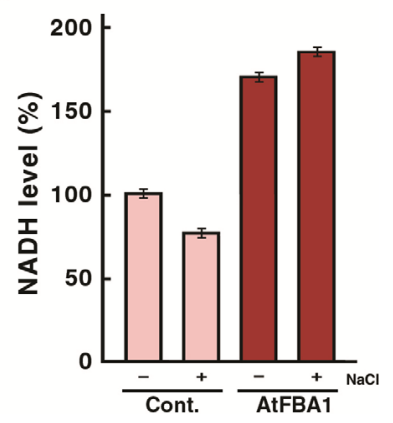

Fig. 2 Expression of AtFBA1 maintains aldolase activity and NADH levels under salt stress in vivo. Yeast were transformed with the empty vector (pYES2) containing the full-length AtFBA1. (A) Aldolase activity was examined in crude extracts after treatment with or without $\mathrm{NaCl}$ as described in the Materials and Methods. (B) NADH levels were detected from (A). The supernatant (50 $\mu \mathrm{g}$ protein) was used to estimate aldolase activity or NADH levels according to the Materials and Methods

aldolase activity decreases under stress conditions, this decrease is complemented by expression of AtFBA1 in yeast.

AtFBA1 transcript levels are specifically reduced by abiotic stress and $A t F B A 1$ is cytosolic in plants

To characterize AtFBA1 in plants, AtFBA1 expression was monitored under various stress conditions. Total RNA was extracted from Arabidopsis seedlings that had been treated with $\mathrm{NaCl}, \mathrm{KCl}$, or the phytohormone ABA. Northern blot analysis was performed using the full-length $A t F B A 1$ as a probe. AtFBA1 transcripts were dramatically reduced following treatment with $\mathrm{NaCl}, \mathrm{KCl}$ and exogenously applied $\mathrm{ABA}$ (Fig. 3). To analyze the tissue-specific AtFBAl expression in plants, semi-quantitative RT-PCR was performed using gene specific primers. Based on the results, AtFBAl is expressed in all plant tissues including roots, stems, leaves, and flowers (Fig. 3).

The subcellular localization of AtFBA1 in Arabidopsis was investigated using green fluorescent protein (GFP). The fulllength AtFBA1 gene was cloned into the p326GFP vector to produce a chimeric AtFBA1:GFP, and the construct was introduced into protoplasts isolated from wild-type Arabidopsis seedlings. The subcellular distribution of AtFBA1:GFP was observed as a green fluorescent signal in the cytosol (Fig. 4). Protoplasts transformed with GFP alone also produced green fluorescent signals in the cytosol. These expression pattern analyses and subcellular localization results indicate that AtFBA1 functions in all plant tissues as a glycolytic enzyme. This enzyme is present in the cytosol and may be negatively regulated in the presence of environmental

A

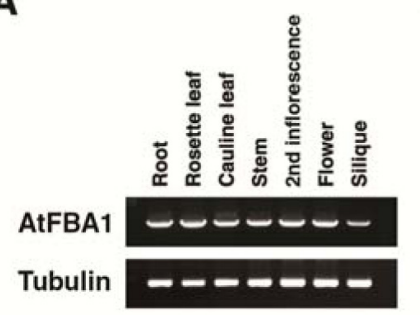

B

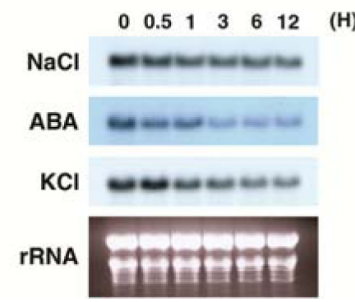

Fig. 3 AtFBA1 transcript levels were decreased by abiotic stress. (A) Tissue specificity of AtFBA1. Total RNA was prepared from each tissue and semi-quantitative reverse transcription PCR (RTPCR) was performed using AtFBA1 specific primers. (B) Total RNA was prepared from 2-week-old Arabidopsis seedlings treated with $\mathrm{NaCl}(100 \mathrm{mM}), \mathrm{ABA}(10 \mu \mathrm{M})$, or $\mathrm{KCl}(100 \mathrm{mM})$ for the indicated times. Equal loading for each lane was confirmed by staining the gels with ethidium bromide (lower). RNA blots were probed with ${ }^{32} \mathrm{P}$-labeled $A t F B A 1$
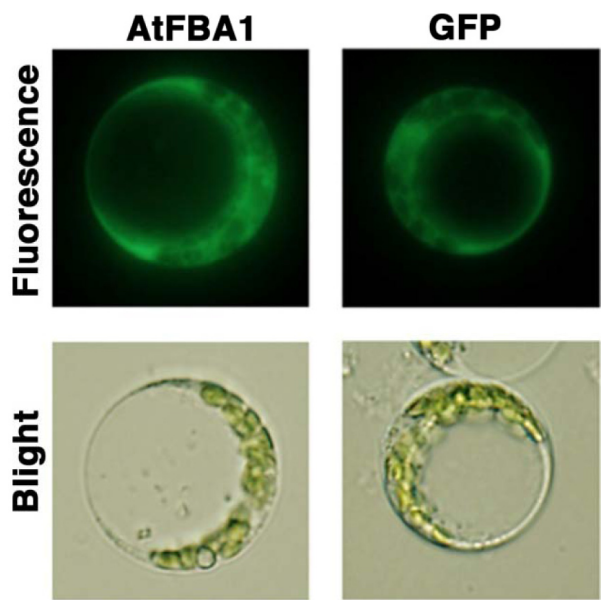

Fig. 4 Subcellular localization of AtFBA1. Protoplasts prepared from Arabidopsis seedlings were transformed with AtFBA1:GFP. The transformed protoplasts were examined by fluorescence microscopy at 12 and $24 \mathrm{~h}$ after transformation

stress in plants.

The AtFBA1 knock-out mutant exhibits sensitivity to abiotic stress

To investigate the biological function of AtFBA1 in plants, a knock-out line containing a single T-DNA insertion in the AtFBA1 locus was identified (atfbal-1, SALK_124383) by searching the Arabidopsis Biological Resource Center. Homozygous plants were screened via PCR and RT-PCR using specific primers. The T-DNA insertion occurs in the third exon of the AtFBA1 locus, resulting in the complete loss of AtFBAl expression in the atfbal-1 mutant (Fig. 5). To observe the phenotype of atfbal-1 under stress conditions, a germination assay and root elongation assay were conducted. 


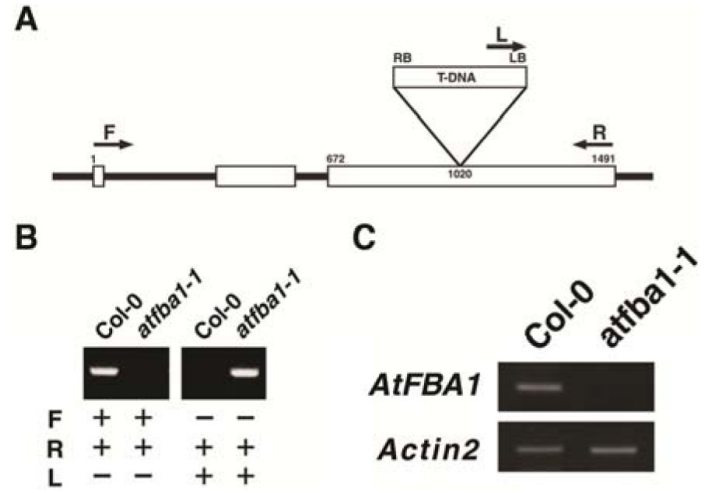

Fig. 5 The T-DNA is inserted in the $3^{\text {rd }}$ exon of the AtFBAl locus. (A) The diagram illustrates the location of the T-DNA insertion in AtFBA1 (At3g52930). Exons (opened boxes) were deduced from the cDNA sequence. The open triangle indicates the T-DNA insertion site. (B) Diagnostic PCR analysis of the T-DNA insertion in wild-type (Col-0) and atfbal-1 using forward (F), reverse (R) and LB (L) primers. (C) Transcript abundance of $A t F B A 1$ in wild-type and atfbal plants as determined by RT-PCR.
To test germination frequency, seeds of wild-type and knockout plants were kept in a cold room $\left(4^{\circ} \mathrm{C}\right)$ for 3 days. Seeds were then sown on $1 / 2$ MS medium with or without ABA treatment. The germination rate of atfbal-1 was minimally affected in comparison to wild-type seeds sown on $1 / 2 \mathrm{MS}$ medium. More than $80 \%$ of the atfbal-1 seeds germinated. After 2 days, the germination rate of atfbal-1 seeds significantly decreased to approximately $40 \%$. By contrast, more than $80 \%$ of the wild-type seeds germinated under conditions of ABA stress (Fig. 6). The germination rate of atfbal-1 seeds was also severely delayed by $\mathrm{NaCl}$ and $\mathrm{KCl}$ treatment when compared to wild-type seeds (Fig. 6).

Next, to characterize the sensitive seedling phenotype of atfbal-1, a root elongation assay was performed under stress conditions. Wild-type and atfbal-1 seeds were grown for 5 days in a vertical orientation and then transferred to $1 / 2$ MS medium containing $\mathrm{NaCl}, \mathrm{KCl}$, or mannitol. After 7
A

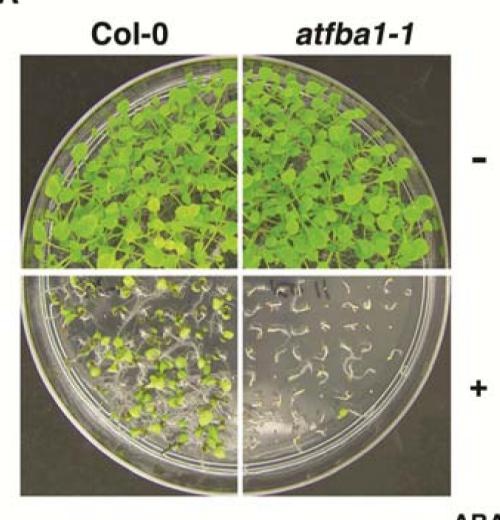

B

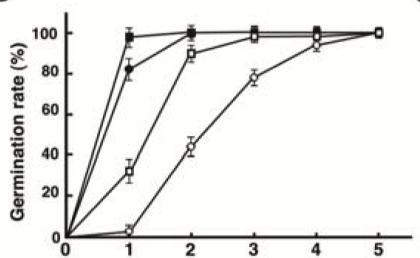

D

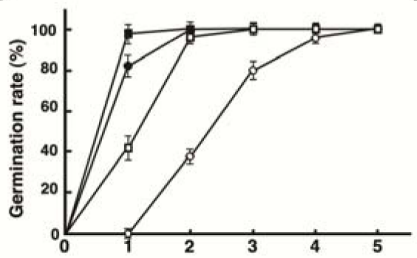

C

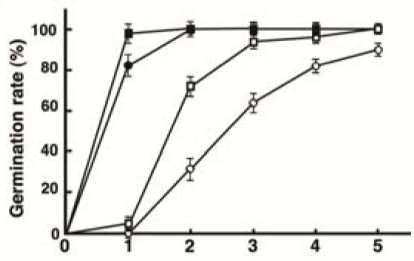

E

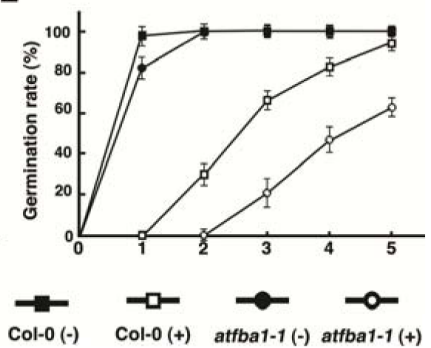

Fig. 6 Germination of atfbal-1 seeds was delayed by abiotic stress. (A) Seedlings of wild type (Col-0) and atfbal-1 were grown under long day conditions in medium containing ABA. Plates were photographed after incubation in a growth chamber for 5 days. (B), (C), and (D) Wild-type (Col-0, squares) and mutant (atfba1-1, circles) seeds were sown on medium with (open) or without (filled) ABA (B), $\mathrm{NaCl}(\mathrm{C})$, and $\mathrm{KCl}$ (D) after 4 days of cold treatment as described in the Materials and Methods. Germination kinetics were determined by calculating the percentage of germination over time. "Germination" in this study refers to the appearance of a radicle of at least $1 \mathrm{~mm}$. Data represent typical data of three independent experiments $(\mathrm{n}=100)$
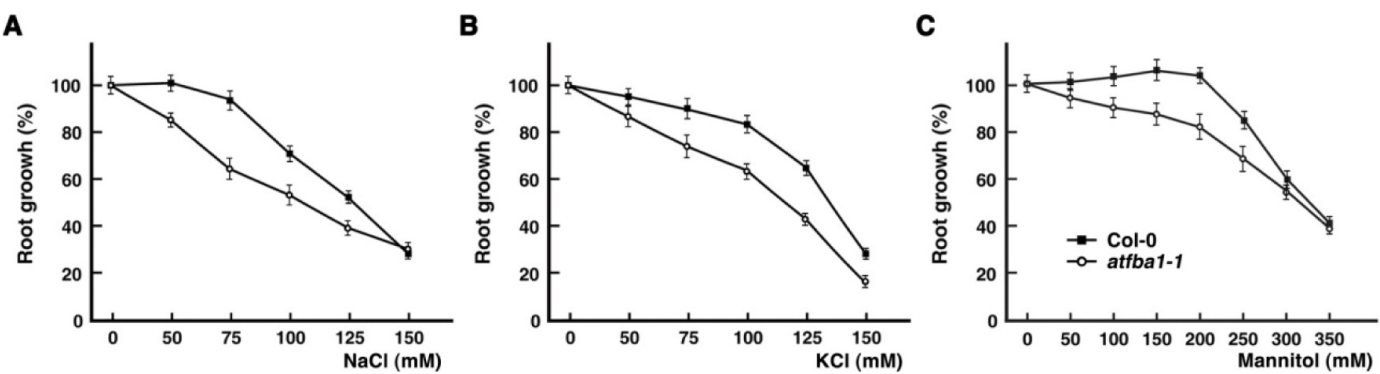

Fig. 7 atfbal-1 is sensitive to abiotic stress. Root growth of Col-0 and atfbal-1 seedlings after treatment with $\mathrm{NaCl}$ (A), $\mathrm{KCl}$ (B), and mannitol (C). Seven-day-old seedlings were transferred to MS medium supplemented with or without each stress treatment. Root growth was measured after 7 days $(n=12)$ 
days, the root lengths of the wild-type and atfbal-1 seedlings were measured. Under normal growth conditions, the root growth of the atfbal-1 mutant was partially retarded. Wildtype root growth was not affected until the $\mathrm{NaCl}$ concentration reached $75 \mathrm{mM}$, and root elongation was disrupted at $100 \mathrm{mM} \mathrm{NaCl}$. The root elongation of atfbal-1 seedlings was inhibited at $50 \mathrm{mM} \mathrm{NaCl}$ and root lengths were reduced to approximately $50 \%$ at $100 \mathrm{mM} \mathrm{NaCl}$ treatment (Fig. 7). Under $\mathrm{KCl}$ and mannitol stress conditions, the atfbal-1 seedlings exhibited similar root growth inhibition in a dosagedependent manner (Fig. 7). These results suggest that AtFBA1 plays a role in the stress tolerance of yeast and plants.

\section{Discussion}

Glycolysis is a ubiquitous primary metabolic pathway in plants. Several glycolytic enzymes, including hexokinase and fructose-1,6-bisphosphate aldolase, contribute to the oxidation of hexoses for the generation of reducing power and pyruvate, as well as to provide precursors for anabolism (Munoz-Bertomeu et al. 2011). However, little is known about the relationship between glycolytic proteins and stress tolerance in plants. This study presents evidence that AtFBA1 maintains cellular aldolase activity and NADH levels under abiotic stress. Based on this evidence, AtFBA1 is proposed to play a role in the stress tolerance of yeast and plants. Plant Class I fructose-1,6-bisphosphate aldolases (FBAs) are grouped into the Class-I type enzymes, forming a Schiff base with the substrate as an intermediate (Lorentzen et al., 2004). These enzymes are found in both the chloroplasts and the cytosol (Lorentzen et al. 2004). Plant chloroplast FBAs mainly function in the generation of metabolites for starch biosynthesis via the Calvin Cycle (Lorentzen et al. 2004). However, cytosolic FBA functions in glycolysis and gluconeogenesis in both source and sink tissues. AtFBAl shares a high degree of sequence identity with Class-I type plant FBAs from rice, maize and spinach. The purified recombinant AtFBA1 exhibits Michaelis-Menten kinetics for FBP in the catabolic (aldol cleavage) direction in a coupled assay system (data not shown). Additionally, the subcellular distribution of AtFBA1:GFP is cytosolic of plant cells (Fig. 4). Therefore, AtFBA1 is assumed to be a functional cytosolic Class I type aldolase in Arabidopsis. Metabolism is a complex non-linear pathway that interacts with many other processes. Photosynthesis is inhibited by decreased expression levels or reduced enzymatic activity of the glycolytic enzymes. Additionally, the levels of sugars and starch are altered and plant growth is compromised.
Genetic analysis has revealed that the glucose response interacts with other plant hormones including ABA. The glucose responsive expression of $A B I 4$ and $A B I 5$ is absent in the glucose insensitive mutants gin 1, gin5, and gin6 (Arenas-Huertero et al. 2000). Yamada et al. demonstrated that $A l d P 2$ is up-regulated and $A l d P 1$ is down-regulated by salt treatment in the Nicotiana species $N$. excelsior and $N$. arentsii (Yamada et al. 2000). Sesuvium portulacastrum $S p F B A$, a gene from a seashore mangrove plant, accumulates in response to high salinity and ABA treatment (Fan et al. 2009). Recently, Gong et al. provided transcriptional profiles of drought-responsive genes in tomato. Among them, fructosebisphosphate aldolase was down-regulated under drought stress conditions (Gong et al. 2010). In this study, the transcript abundance of $A t F B A 1$ was negatively regulated by ABA treatment and stress conditions (Fig. 3). Under salt stress, the aldolase activity and NADH level are decreased in yeast cells. However, when AtFBAl is expressed in yeast, the aldolase activity and NADH level are increased with or without salt stress (Fig. 2). These results indicate that the activity of some glycolytic enzymes, such as aldolase, are affected by stress conditions. At a minimum, these activities are regulated at the transcriptional level in yeast and plants.

GAPDH, a glycolytic enzyme, is one of the most abundant soluble proteins. GAPDH is encoded by three unlinked genes designated $T D H 1, T D H 2$, and $T D H 3$ in S. cerevisiae. Strains lacking $T D H 3$ ( $t d h 3$ and $t d h 1 t d h 3$ strains) exhibit extreme sensitivity to $\mathrm{H}_{2} \mathrm{O}_{2}$ compared to wild-type controls. The strain lacking both $T D H 1$ and $T D H 3$ is the most sensitive (Grant et al. 1999). Overexpression of a cyanobacterial fructose-1,6-/sedoheptulose 1,7-bisphosphatase enhances photosynthesis and growth in tobacco (Miyagawa et al. 2001). Fructose-1,6-bisphosphate aldolase (FBA1) from S. cerevisiae is single copy gene, and deletion mutants of this gene exhibit growth defects (Compagno et al. 1991). Inhibition of chloroplastic fructose-1,6-phosphatase in tomato leads to decreased fruit size and small changes in carbohydrate metabolism (Obiadalla-Ali et al. 2004). Konishi et al. also suggested that fructose-bisphosphate aldolase is regulated by gibberellins, and the root growth of antisense transgenic rice was repressed (Konishi et al. 2004). Haake et al. showed that decreased aldolase activity in antisense transgenic potato results in alterations in sugar and starch levels, and inhibition of plant growth (Haake et al. 1998). Expression of AtFBA1 rescues the salt-sensitive phenotype of the $c n b \Delta$ yeast mutant (Fig. 1). atfbal shows delayed germination and root elongation under abiotic stress conditions (Fig. 6 and 7). Taken together, glycolysis and gluconeogenesis are proposed to be sensitive to abiotic stresses such as high salinity. 
Furthermore, expression of AtFBA1 maintains glycolysis flux under stress conditions.

\section{Acknowledgments}

This work was supported by a grant from the Cooperative Research Program for National Academy of Agricultural Science (PJ00665404), and the Next-Generation BioGreen 21 Program (PJ0080420), Rural Development Administration, Republic of Korea. Shin D. and Moon S.J. were supported by the Postdoctoral Course Program of the National Academy of Agricultural Science, Rural Development Administration, Republic of Korea.

\section{References}

Arenas-Huertero F., Arroyo A., Zhou L., Sheen J., and Leon P. (2000) Analysis of Arabidopsis glucose insensitive mutants, gin5 and gin6, reveals a central role of the plant hormone ABA in the regulation of plant vegetative development by sugar. Genes Dev 14:2085-2096

Baier M., Hemmann G., Holman R., Corke F., Card R., Smith C., Rook F. and Bevan M.W. (2004) Characterization of mutants in Arabidopsis showing increased sugar-specific gene expression, growth, and developmental responses. Plant Physiol 134:81-91

Chew Y.H., and Halliday K.J. (2011) A stress-free walk from Arabidopsis to crops. Curr Opin Biotechnol 22:281-286

Cho Y.H. and Yoo S.D. (2011) Signaling role of fructose mediated by FINS1/FBP in Arabidopsis thaliana. PLoS Genet 7, e1001263

Cho Y.H., Yoo S.D. and Sheen J. (2006) Regulatory functions of nuclear hexokinase1 complex in glucose signaling. Cell 127: 579-589

Compagno C., Ranzi B.M. and Martegani E. (1991) The promoter of Saccharomyces cerevisiae FBA1 gene contains a single positive upstream regulatory element. FEBS Lett 293: 97-100

Fan W., Zhang Z. and Zhang Y. (2009) Cloning and molecular characterization of fructose-1,6-bisphosphate aldolase gene regulated by high-salinity and drought in Sesuvium portulacastrum. Plant Cell Rep 28:975-984

Finkelstein R.R. and Gibson S.I. (2002) ABA and sugar interactions regulating development: cross-talk or voices in a crowd? Curr Opin Plant Biol 5:26-32

Gong P., Zhang J., Li H., Yang C., Zhang C., Zhang X., Khurram Z., Zhang Y., Wang T., Fei Z. and Ye Z. (2010) Transcriptional profiles of drought-responsive genes in modulating transcription signal transduction, and biochemical pathways in tomato. $\mathrm{J}$ Exp Bot 61:3563-3575

Grant C.M., Quinn K.A. and Dawes I.W. (1999) Differential protein S-thiolation of glyceraldehyde-3-phosphate dehydrogenase isoenzymes influences sensitivity to oxidative stress. Mol Cell Biol 19:2650-2656

Haake V., Zrenner R., Sonnewald U. and Stitt M. (1998) A moderate decrease of plastid aldolase activity inhibits photosynthesis, alters the levels of sugars and starch, and inhibits growth of potato plants. Plant J 14:147-157

Halford N.G. and Hey S.J. (2009) Snf1-related protein kinases (SnRKs) act within an intricate network that links metabolic and stress signalling in plants. Biochem J 419:247-259

Jang J.C., Leon P., Zhou L. and Sheen J. (1997) Hexokinase as a sugar sensor in higher plants. Plant Cell 9:5-19

Jin X.F., Xiong A.S., Peng R.H., Liu J.G., Gao F., Chen J.M. and Yao, Q.H. (2010) OsAREB1, an ABRE-binding protein responding to $\mathrm{ABA}$ and glucose, has multiple functions in Arabidopsis. BMB Rep 43:34-39

Konishi H., Yamane H., Maeshima M. and Komatsu S. (2004) Characterization of fructose-bisphosphate aldolase regulated by gibberellin in roots of rice seedling. Plant Mol Biol 56: 839-848

Kushwah S., Jones A.M. and Laxmi A. (2011) Cytokinin interplay with ethylene, auxin, and glucose signaling controls Arabidopsis seedling root directional growth. Plant Physiol 156:1851-1866

Lorentzen E., Siebers B., Hensel R. and Pohl E. (2004) Structure, function and evolution of the Archaeal class I fructose-1,6bisphosphate aldolase. Biochem Soc Trans 32:259-263

Melcher K., Ng L.M., Zhou X.E., Soon F.F., Xu Y., Suino- Powell K.M., Park S.Y., Weiner J.J., Fujii H., Chinnusamy V., Kovach A., Li J., Wang Y., Peterson F.C., Jensen D.R., Yong E.L., Volkman B.F., Cutler S.R., Zhu J.K. and Xu H.E. (2009) A gate-latch-lock mechanism for hormone signalling by abscisic acid receptors. Nature 462:602-608

Miyagawa Y., Tamoi M. and Shigeoka S. (2001) Overexpression of a cyanobacterial fructose-1,6-/sedoheptulose-1,7-bisphosphatase in tobacco enhances photosynthesis and growth. Nat Biotechnol 19:965-969

Moore B., Zhou L., Rolland F., Hall Q., Cheng W.H., Liu Y.X., Hwang I., Jones T. and Sheen J. (2003) Role of the Arabidopsis glucose sensor HXK1 in nutrient, light, and hormonal signaling. Science 300:332-336

Munoz-Bertomeu J., Anoman A.D., Toujani W., Cascales-Minana B., Flores-Tornero M. and Ros R. (2011) Interactions between abscisic acid and plastidial glycolysis in Arabidopsis. Plant Signal Behav 6:157-159

Nishimura N., Sarkeshik A., Nito K., Park S.Y., Wang A., Carvalho P.C., Lee S., Caddell D.F., Cutler S.R., Chory J., Yates J.R. and Schroeder J.I. (2010) PYR/PYL/RCAR family members are major in-vivo ABI1 protein phosphatase 2C-interacting proteins in Arabidopsis. Plant J 61:290-299

Obiadalla-Ali H., Fernie A.R., Lytovchenko A., Kossmann J. and Lloyd J.R. (2004) Inhibition of chloroplastic fructose 1,6bisphosphatase in tomato fruits leads to decreased fruit size, but only small changes in carbohydrate metabolism. Planta 219:533-540

Park S.Y., Fung P., Nishimura N., Jensen D.R., Fujii H., Zhao Y., 
Lumba S., Santiago J., Rodrigues A., Chow T.F., Alfred S.E., Bonetta D., Finkelstein R., Provart N.J., Desveaux D., Rodriguez P.L., McCourt P., Zhu J.K., Schroeder J.I., Volkman B.F. and Cutler S.R. (2009) Abscisic acid inhibits type $2 \mathrm{C}$ protein phosphatases via the PYR/PYL family of START proteins. Science 324:1068-1071

Price J., Li T.C., Kang S.G., Na J.K. and Jang J.C. (2003) Mechanisms of glucose signaling during germination of Arabidopsis. Plant Physiol 132:1424-1438

Quan R., Hu S., Zhang Z., Zhang H. and Huang R. (2010) Overexpression of an ERF transcription factor TSRF1 improves rice drought tolerance. Plant Biotechnol J 8:476-488

Rao S.R., Ford K.L., Cassin A.M., Roessner U., Patterson J.H. and Bacic, A. (2010) Proteomic and metabolic profiling of rice suspension culture cells as a model to study abscisic acid signaling response pathways in plants. J Proteome Res 9: 6623-6634

Shin D., Koo Y.D., Lee J., Lee H.J., Baek D., Lee S., Cheon C.I., Kwak S.S., Lee S.Y. and Yun D.J. (2004) Athb-12, a homeobox-leucine zipper domain protein from Arabidopsis thaliana, increases salt tolerance in yeast by regulating sodium exclusion. Biochem Biophys Res Commun 323:534-540

Shin D., Moon S.J., Han S., Kim B.G., Park S.R., Lee S.K., Yoon H.J., Lee H.E., Kwon H.B., Baek D., Yi B.Y. and Byun M.O. (2011) Expression of StMYB1R-1, a novel potato single MYB-like domain transcription factor, increases drought tolerance. Plant Physiol 155:421-432

Skirycz A., and Inze D. (2010) More from less: plant growth under limited water. Curr Opin Biotechnol 21:197-203

Wang R.S., Pandey S., Li, S., Gookin T.E., Zhao Z., Albert R. and Assmann, S.M. (2011) Common and unique elements of the ABA-regulated transcriptome of Arabidopsis guard cells. BMC Genomics 12:216

Yamada S., Komori T., Hashimoto A., Kuwata S., Imaseki H. and Kubo T. (2000) Differential expression of plastidic aldolase genes in Nicotiana plants under salt stress. Plant Sci 154: 61-69 